\title{
A NOMEAÇÃO SERIADA RÁPIDA EM ESCOLARES \\ COM E SEM QUEIXAS DE PROBLEMAS DE APRENDIZAGEM EM ESCOLA PÚBLICA E PARTICULAR
}

\author{
The rapid serial naming in students with and without complaints \\ of learning problems in public and private schools
}

Lorena Gabrielle Ribeiro Bicalho ${ }^{(1)}$, Luciana Mendonça Alves ${ }^{(2)}$

\begin{abstract}
RESUMO
Objetivo: investigar a nomeação seriada rápida em crianças de uma escola pública e uma privada, com e sem queixas de problemas escolares. Métodos: participaram do estudo 137 crianças dos gêneros masculino e feminino, com idade entre 7 e 11 anos, da cidade de Belo Horizonte, matriculadas no ensino fundamental, entre o $2^{\circ}$ e $\circ 5^{\circ}$ ano. Foi aplicado o Teste de Nomeação Automatizada Rápida (Rapid Automatized Naming - RAN) que avaliou o tempo gasto pelo participante para nomear uma série de estímulos visuais familiares: cores, letras, dígitos e objetos - o mais rápido possível. Resultados: os resultados deste estudo evidenciaram que o desempenho de alunos de escola particular comparado ao de alunos de escola pública é superior e que há uma diferença estatisticamente significante entre sujeitos com queixas de problemas de aprendizagem comparado aos sem queixas. Conclusão: o presente estudo sinaliza que a capacidade de nomeação seriada pode ser considerada como uma das habilidades fundamentais para o bom desempenho em leitura. Sendo assim, há necessidade de continuidade de pesquisas que estabeleçam a relação dessa habilidade da linguagem com outras habilidades de leitura e escrita para verificação do impacto direto da nomeação seriada rápida no processo de aprendizagem da leitura.
\end{abstract}

DESCRITORES: Aprendizagem; Criança; Leitura; Medição de Velocidade

\section{INTRODUÇÃO}

$\mathrm{Na}$ atual realidade brasileira, o número de crianças com dificuldades de aprendizagem tem crescido de forma acelerada. As principais queixas referemse às questões de leitura, escrita e cálculo matemático. Vários fatores interferem no processo de aprendizagem da leitura e escrita como, por exemplo, a adequada instrução educacional e a própria estrutura linguístico-cognitiva do escolar. $O$ desen-

(1) Fonoaudióloga; Clínica Parlare, Fonoaudiologia e Reabilitação Auditiva, Belo Horizonte, MG, Brasil.

(2) Fonoaudióloga; Docente do curso de Graduação em Fonoaudiologia do Centro Universitário Metodista Izabela Hendrix, Belo Horizonte, MG, Brasil; Docente do curso de PósGraduação da FEAD - Centro de Gestão Empreendedora e do CEFAC - Pós-Graduação em Saúde e Educação, Belo Horizonte, MG, Brasil; Doutora em Estudos Linguísticos pela Universidade Federal de Minas Gerais.

Conflito de interesses: inexistente volvimento dessa aprendizagem tão complexa varia de criança para criança, podendo ocorrer em tempos diferentes de acordo com cada uma ${ }^{1}$.

Aprender a ler não é um processo natural. Contrariamente à linguagem oral a leitura não emerge naturalmente da interação com os pais e os outros adultos, por mais estimulante que seja o meio cultural $^{2}$.

No aprendizado da linguagem escrita existe uma combinação dos fenômenos biológicos e ambientais, envolvendo a integridade motora, a integridade sensório-perceptual e a integridade sócio-emocional (possibilidades reais que o meio oferece em termos de quantidade, qualidade e frequência de estímulos) ${ }^{3}$.

Ler e escrever, assim como as demais atividades humanas, demandam tempo e prática para se tornarem processos automáticos ${ }^{4}$.

Nas últimas três décadas, as pesquisas têm dado ênfase à relação entre os processamentos 
perceptuais, linguísticos e cognitivos envolvidos na leitura ${ }^{5}$. Uma das habilidades do processamento fonológico mais estudada internacionalmente no período citado foi a nomeação rápida ${ }^{6}$. A partir de pesquisas, foi possível definir padrões de normalidade em diversas modalidades de tarefas de nomeação rápida 7 .

Atualmente já se sabe que o cérebro possui áreas específicas para várias funções e que a região occipital-temporal é a área onde se processa o reconhecimento visual das palavras, e onde se realiza a leitura rápida e automática. Quanto mais automaticamente for feita a ativação dessa área, mais eficiente é o processo de leitura. Os leitores eficientes utilizam esse percurso rápido e automático para ler as palavras. Ativam intensamente os sistemas neurológicos que envolvem a região parietal-temporal e a occipital-temporal e conseguem ler as palavras instantaneamente (em menos de 150 milésimos de segundo) ${ }^{8}$.

Como consequência destes estudos é possível a aplicação de avaliações que possibilitam aos pesquisadores verificar o uso de habilidades do processamento fonológico, como a consciência fonológica, a memória de trabalho e a nomeação rápida em populações de bons leitores ou com alterações no processo de aprendizagem na leitura ${ }^{9}$.

Outros estudos mostraram que crianças com queixas de problemas de aprendizagem também podem vir a apresentar problemas de acesso ao léxico decorrentes de diferentes níveis do processamento da informação e que a velocidade de nomeação de estímulos encontra-se diretamente ligada à velocidade de acesso a memória de curto prazo e a nomeação fonológica, influenciando assim o desenvolvimento da escrita e da leitura ${ }^{10}$.

A partir dessas pesquisas vários estudos internacionais vêm documentando o baixo desempenho de maus leitores em nomeação rápida. No Brasil há poucos estudos nesta área deixando uma ampla lacuna na literatura.

A habilidade de processar símbolos visuais rapidamente é usualmente avaliada por intermédio de tarefas de nomeação automatizada rápida. Avalia-se, nessas tarefas, o tempo gasto pelo indivíduo para nomear uma série de estímulos visuais familiares: cores, letras, dígitos e objetos - o mais rápido possível. Uma vez que a rapidez é também um fator importante para a leitura textual fluente, não é surpreendente que o desempenho em tarefas de nomeação automatizada rápida correlacione-se com o desempenho em medidas de leitura fluente de textos ${ }^{11}$.

O tempo que a criança leva para processar uma informação visual escrita e/ou uma imagem pode apontar para dificuldades na nomeação e na leitura e conduz a hipóteses sobre desenvolvimento, tanto de linguagem como cognitivo, afinal quanto maior a competência em se reconhecer palavras escritas de modo rápido e acurado, maiores os recursos cognitivos disponíveis para a tarefa de compreensão de leitura ${ }^{12}$.

Leitores proficientes são capazes de ler palavras a uma velocidade bastante rápida, chegando a 300 palavras por minuto, ou cinco palavras por segundo ${ }^{13}$.

O teste RAN é um teste de fácil aplicação e de grande valia, pois funciona como uma ferramenta para diagnosticar problemas potenciais da leitura, permitindo a detecção precoce e a devida intervenção ${ }^{14}$.

Busca-se, desta forma, contribuir para a escassa literatura nacional sobre o assunto e ampliar o conhecimento fonoaudiológico sobre o teste RAN e sua aplicação na avaliação dos transtornos de aprendizagem. Com esta pesquisa caminhamos na direção de prover parâmetros de resposta esperados para a população mineira.

Assim sendo, o objetivo deste estudo é verificar o desempenho, no teste de nomeação rápida automatizada (RAN), de escolares com queixas de dificuldades de aprendizagem, e escolares sem histórico de dificuldade de aprendizagem, que lêem conforme sua idade e escolaridade, em escolas públicas e particulares.

\section{MÉTODOS}

Tratou-se de um estudo observacional analítico com delineamento caso controle.

Participaram desta pesquisa 137 escolares dos gêneros masculino e feminino, do $2^{\circ}$ ao $5^{\circ}$ ano do Ensino Fundamental de uma escola da rede pública e outra da rede particular de ensino de Belo Horizonte.

A escolha por esta faixa de escolaridade $\left(2^{\circ}\right.$ ao $5^{\circ}$ ano) foi feita por ser um período em que o estudante já passou da fase alfabética de leitura e está plenamente inserido no contexto escolar, época em que normalmente são diagnosticados os problemas de aprendizagem.

Foram entregues em média 300 termos de consentimento com uma média de $50 \%$ de devolução dos mesmos. Obtiveram-se em torno de 37 alunos por série. Uma vez que nesta pesquisa constaram alunos de escola particular e pública, obtiveramse 150 sujeitos. Mas considerando os critérios de exclusão que serão especificados a seguir, restaram 137 sujeitos que preencheram os critérios para a presente pesquisa. Optou-se por uma escola pública e outra particular para que se pudesse investigar sujeitos de ambas as realidades sócio- 
educacionais. O critério de seleção das escolas foi o interesse apresentado pelas mesmas para a realização da pesquisa, com disponibilidade de uma sala à parte, longe de ruídos e movimentação que pudessem interferir na coleta.

Primeiramente, os responsáveis pelas crianças participantes da pesquisa foram orientados e receberam o Termo de Consentimento Livre e Esclarecido. Somente aquelas crianças cujos pais aceitaram participar da pesquisa, assinaram e entregaram o Termo de Consentimento aos pesquisadores, participaram deste estudo.

A divisão dos participantes em grupos de escolares com e sem queixas de dificuldades de aprendizagem realizou-se por meio da aplicação de um questionário destinado ao responsável da criança ${ }^{15}$ e outro ao professor ${ }^{16}$. Por meio destes questionários foi possível obter informações dos pais referentes a possíveis dificuldades apresentadas pela criança, dados sobre o desenvolvimento global do participante, sobre os aspectos referentes ao aprendizado da fala e da linguagem, ao aprendizado escolar e quanto aos antecedentes de doenças. Já o questionário direcionado aos professores permitiu classificar os alunos, por critérios objetivos, em três categorias: a) alunos que leem bem; b) alunos que leem mais ou menos; c) alunos que leem mal.

A partir desta lista classificatória, foram escolhidos os sujeitos classificados como bons leitores, de modo a compor o número amostral de 97 estudantes sem queixas de alterações de linguagem oral e escrita, e os alunos que leem mal compuseram o grupo das 40 crianças com queixas. Os alunos classificados como alunos que leem mais ou menos foram direcionados para um dos dois grupos descritos acima utilizando como critério o questionário dos pais. Os casos duvidosos tiveram sua classificação discutida pessoalmente com os professores.

Os critérios de inclusão na pesquisa foram os escolares estarem devidamente matriculados na escola pesquisada, entre o $2^{\circ}$ e $\circ 5^{\circ}$ ano do Ensino Fundamental, terem o termo de consentimento assinado pelo responsável, não apresentarem problema de saúde que direta ou indiretamente interfira no desenvolvimento da linguagem indicado pelo questionário dirigido aos pais. Os critérios de exclusão foram: crianças cujos pais não assinaram o termo de consentimento, que apontaram a existência de algum quadro patológico não relacionado aos distúrbios da linguagem escrita no questionário, e que apresentaram idade superior a idade determinada para a sua série.

Todas as crianças foram submetidas ao teste RAN ${ }^{7}$ que consiste de quatro conjuntos de estímulos, os quais aparecem impressos em 5 fileiras de
10 itens cada. Os quatro conjuntos de estímulos são: 1) cores (vermelho, amarelo, verde, azul, preto); 2) letras minúsculas $(\mathrm{a}, \mathrm{d}, \mathrm{o}, \mathrm{s}, \mathrm{p})$; 3 ) números $(2,4,6$, $7,9)$; 3) e, 4) desenhos de objetos comuns (guardachuva, tesoura, pente, relógio, chave). Os estímulos em cada conjunto foram apresentados em ordem aleatória, em um total de 10 vezes cada um. O participante foi instruído a nomear os estímulos visuais apresentados o mais rápido possível. O tempo foi registrado com cronômetro.

A aplicação do Teste de Nomeação Automatizada Rápida (RAN) durou 10 minutos. Todos os procedimentos foram realizados na própria escola, individualmente, em uma sala à parte, em horário não prejudicial à aprendizagem do conteúdo curricular.

Os escolares foram pareados por idade e escolaridade e subdivididos em quatro grupos:

- Grupo 1 (G1): composto por escolares do $2^{\circ}$ ano com idade entre 7 e 8 anos.

- Grupo 2 (G2): composto por escolares do $3^{\circ}$ ano com idade entre 8 e 9 anos.

- Grupo 3 (G3): composto por escolares do $4^{\circ}$ ano com idade entre 9 e 10 anos.

- Grupo 4 (G4): composto por escolares do $5^{\circ}$ ano com idade entre 10 e 11 anos.

A pesquisa foi aprovada pelo Comitê de Ética em Pesquisa (CEP) do Centro Universitário Metodista Izabela Hendrix, de acordo com o parecer $n^{\circ} 214 / 2008$.

O suporte para o tratamento estatístico dos dados foi feito da seguinte forma: foram calculados as médias, o respectivo desvio padrão e o coeficiente de variação para cada variável estudada, em cada grupo pesquisado. Em seguida, foi conduzida uma comparação entre os grupos e, para cada comparação, foi investigado se a diferença entre as médias é estatisticamente significante, utilizandose o teste t de Student. Os resultados estatisticamente significantes foram assinalados por asterisco $\left(^{*}\right)$. Adotou-se o nível de significância de 5\% $(0,050)$ para a aplicação dos testes estatísticos, ou seja, quando o valor da significância calculada (p) for menor do que $5 \%(0,050)$ observa-se uma diferença "estatisticamente significante".

\section{RESULTADOS}

Fizeram parte deste estudo 137 escolares, sendo que 97 estudantes não apresentavam queixas de alterações de linguagem oral e escrita, e 40 apresentavam tais queixas. Dos 137 escolares, 69 eram alunos da rede privada de ensino e 68 da rede pública de ensino, 75 estudantes eram do gênero feminino e 62 do gênero masculino. 
A Tabela 1 apresenta as médias de tempo obtidas pelos sujeitos estudados do G1, G2, G3 e G4, quanto à velocidade de nomeação de cores, letras, dígitos e números, fazendo uma comparação entre os alunos da escola pública e da escola particular.

Com a aplicação do teste t de Student, foi possível verificar em alguns dos subtestes do RAN, diferenças estatisticamente significantes entre os grupos da escola particular (GPA) comparado aos da escola pública (GPU), evidenciando-se melhor desempenho dos alunos do GPA nessa pesquisa. Os sujeitos do G1PA apresentaram um melhor resultado no subteste de cores, quando comparados com os sujeitos do G1PU, que apresentam

Tabela 1 - Distribuição da média, desvio-padrão e p-valor do desempenho dos escolares dos grupos G1, G2, G3 e G4, comparando alunos da escola pública (GPU) e escola particular (GPA) nos subtestes do teste de nomeação automática rápida (RAN)

\begin{tabular}{|c|c|c|c|c|}
\hline & Grupos & Média & Desvio padrão & Significância (P) \\
\hline \multirow{8}{*}{ Cores } & G1PA & 0:00: 52 & 0:00: 08 & \multirow{2}{*}{0,01672 * } \\
\hline & G1PU & 0:01: 00 & 0:00: 05 & \\
\hline & G2PA & 0:00: 44 & 0:00: 08 & \multirow{2}{*}{0,21111} \\
\hline & G2PU & 0:00: 58 & 0:00: 37 & \\
\hline & G3PA & 0:00: 44 & 0:00: 08 & \multirow{2}{*}{0,21807} \\
\hline & G3PU & 0:00: 47 & 0:00: 08 & \\
\hline & G4PA & 0:00: 40 & 0:00: 08 & \multirow{2}{*}{$0,04774^{*}$} \\
\hline & G4PU & 0:00: 45 & 0:00: 09 & \\
\hline & Grupos & Média & Desvio padrão & Significância (P) \\
\hline \multirow{8}{*}{ Letras } & G1PA & 0:00: 27 & 0:00: 05 & \multirow{2}{*}{0,20305} \\
\hline & G1PU & 0:00: 42 & 0:00: 21 & \\
\hline & G2PA & 0:00: 26 & 0:00: 04 & \multirow{2}{*}{$0,02227^{*}$} \\
\hline & G2PU & 0:00: 42 & 0:00: 22 & \\
\hline & G3PA & 0:00: 26 & 0:00: 06 & \multirow{2}{*}{0,01031 * } \\
\hline & G3PU & 0:00: 31 & 0:00: 06 & \\
\hline & G4PA & $0: 00: 21$ & 0:00:05 & \multirow{2}{*}{0,00024 * } \\
\hline & G4PU & 0:00:29 & 0:00:09 & \\
\hline & Grupos & Média & Desvio padrão & Significância (P) \\
\hline \multirow{9}{*}{ Dígitos } & G1PA & 0:00:34 & 0:00:07 & \multirow{2}{*}{0,04334 * } \\
\hline & G1PU & 0:00:45 & 0:00:09 & \\
\hline & G2PA & 0:00:29 & 0:00:06 & \multirow{2}{*}{0,09724} \\
\hline & G2PU & $0: 00: 41$ & 0:00:24 & \\
\hline & G3PA & 0:00:29 & 0:00:07 & \multirow{2}{*}{0,08447} \\
\hline & G3PU & $0: 00: 33$ & $0: 00: 28$ & \\
\hline & G4PA & $0: 00: 24$ & 0:00:04 & \multirow{2}{*}{0,00058 * } \\
\hline & G4PU & 0:00: 31 & 0:00: 07 & \\
\hline & Grupos & Média & Desvio padrão & Significância (P) \\
\hline \multirow{8}{*}{ Objetos } & G1PA & 0:01: 05 & 0:00: 13 & \multirow{2}{*}{0,18999} \\
\hline & G1PU & 0:01: 21 & 0:00: 22 & \\
\hline & G2PA & 0:01: 01 & 0:00: 10 & \multirow{2}{*}{0,14753} \\
\hline & G2PU & 0:01: 15 & 0:00: 30 & \\
\hline & G3PA & 0:01: 03 & 0:00: 14 & \multirow{2}{*}{0,48934} \\
\hline & G3PU & 0:01: 07 & 0:00: 21 & \\
\hline & G4PA & 0:00: 51 & 0:00: 09 & \multirow{2}{*}{0,00253 * } \\
\hline & G4PU & 0:01: 07 & 0:00: 21 & \\
\hline
\end{tabular}

Significância $=p<0,05$

$\mathrm{GPA}=$ Grupo escola Particular; GPU $=$ Grupo escola Pública; $\mathrm{G} 1=2^{\circ}$ ano $/ \mathrm{G} 2=3^{\circ}$ ano $/ \mathrm{G} 3=4^{\circ}$ ano $/ \mathrm{G} 4=5^{\circ}$ ano 
mesma idade e escolaridade, tais resultados foram estatisticamente significantes.

Os sujeitos dos grupos G2PA, G3PA e G4PA apresentaram melhor resultado no subteste de letras, quando comparados aos seus pares da escola Pública, G2PU, G3PU e G4PU, tais resultados foram estatisticamente significantes.

Os sujeitos do G4PA apresentaram melhor desempenho em todos os subtestes do RAN comparados ao G4PU. Em nenhum dos subtestes os sujeitos do GPU apresentaram melhor desempenho que os sujeitos do GPA nessa pesquisa.

Ao dividir os sujeitos pesquisados em dois grandes grupos, sendo analisado, de forma geral, o desempenho dos sujeitos da escola particular comparados com os da escola pública, foi possível observar uma diferença significante no subteste de objetos. Os sujeitos do GPA apresentaram no subteste de objetos uma média de 0:01:00 min e desvio padrão de 0:00:12 seg, os sujeitos do GPU apresentaram média de 0:01:08 min e desvio padrão de 0:00:22 seg. Tais resultados foram estatisticamente significantes $(p=0 ; 008)$.

Durante a análise estatística, o desvio padrão encontrado no GPU foi usualmente maior quando comparado ao GPA (Tabela 1), demonstrando uma maior variabilidade nos valores encontrados, o que nos revela a heterogeneidade dos resultados obtidos para o GPU.

A Tabela 2 apresenta as médias de tempo obtidas pelos sujeitos do G1, G2, G3 e G4 quanto à velocidade de nomeação comparando os sujeitos com queixas de problemas de aprendizagem aos sem queixas. Foram observados resultados estatisticamente significantes nos sujeitos do G3 e G4 nos subtestes de letras, dígitos e objetos, quando comparados indivíduos com queixas ao sem queixas. Nos demais grupos não foram observadas diferenças significantes. Nos subtestes de letras e dígitos foram apresentadas diferenças significantes quando comparados de forma global os alunos com queixas de problemas de aprendizagem aos sem queixas, observado na Tabela 3.

A Tabela 4 apresenta as médias de tempo obtidas pelos sujeitos estudados, quanto à velocidade de nomeação fazendo uma comparação entre o gênero masculino e o gênero feminino. Não foram evidenciadas diferenças estatisticamente significantes quanto ao sexo dos sujeitos.

Na Tabela 5 são apresentados os tempos mínimos e máximos obtidos pelos sujeitos dos grupos G1, G2, G3 e G4 que não apresentaram queixas de problemas de aprendizagem. Nesta tabela não foram apresentados os resultados encontrados para os sujeitos com queixas de problemas de aprendizagem, pois com esta tabela, o objetivo é caminhar no sentido de uma padronização dos possíveis resultados para bons leitores na população mineira.

Quanto aos códigos letras e números, ocorreu diferença estatisticamente significante, sendo que todos os sujeitos pesquisados nomearam com maior velocidade tais códigos quando comparados aos de cores e objetos. A nomeação de cores ocorreu com maior rapidez quando comparada a de objetos $(p<0,05)$.

\section{DISCUSSÃO}

Das 137 crianças pesquisadas, 40 apresentaram relato, dos pais e/ou professores, de dificuldades de aprendizagem. Tais dados são compatíveis com os achados da literatura nacional ${ }^{17}$ que afirma que no Brasil cerca de 30 a $40 \%$ da população que frequenta as primeiras séries do Ensino Fundamental têm algum tipo de dificuldade.

Os resultados deste estudo evidenciaram que o desempenho de alunos de escola particular comparado ao de alunos de escola pública é superior, corroborando os achados da literatura ${ }^{18}$ que evidenciam que o tempo de nomeação rápida depende também do nível sócio-econômico-cultural do indivíduo. Esses resultados, possivelmente, estão relacionados às práticas educativas utilizadas pelos professores das diferentes escolas, ao suporte familiar dado pelos pais com melhores condições sócio-culturais, ao ambiente social mais estimulador (contato precoce com livros, jornais, Internet e outros materiais).

Nos resultados obtidos pelos alunos da escola pública, o desvio padrão encontrado foi usualmente maior quando comparado ao resultado dos alunos da escola particular, demonstrando uma maior variabilidade nos valores encontrados, o que revela a heterogeneidade dos resultados obtidos pelos sujeitos do ensino público. Tal heterogeneidade pode ser decorrente de diferenças no nível sócio-econômico médio de seus alunos, do tipo de estimulação extra-escolar recebida pelo aluno, ambiente familiar desfavorável, problemas emocionais, do nível de motivação para o tipo de tarefa proposta, entre outros ${ }^{19}$.

O tempo de nomeação não variou entre sujeitos do gênero feminino comparado ao gênero masculino, indo contra os achados da literatura ${ }^{7}$ da década de 70 que afirmavam que as meninas eram mais rápidas que os meninos, mas reafirmou os dados da literatura recente ${ }^{10}$.

O tempo de nomeação dos sujeitos com queixa de problemas de aprendizagem comparado aos sem queixas apresentou uma diferença significante, sendo uma questão unânime na literatura 
Tabela 2 - Distribuição da média, desvio-padrão e p-valor do desempenho dos escolares dos grupos G1, G2, G3 e G4, comparando sujeitos com queixas aos sem queixas de transtornos de aprendizagem nos subtestes do teste de nomeação automática rápida (RAN)

\begin{tabular}{|c|c|c|c|c|c|}
\hline & & Grupo & Média & Desvio padrão & Significância $(P)$ \\
\hline \multirow{2}{*}{ Cores } & COM QUEIXA & \multirow{2}{*}{ G1 } & 0:00: 57 & 0:00: 10 & \multirow{2}{*}{0,24953} \\
\hline & SEM QUEIXA & & 0:00: 52 & 0:00: 07 & \\
\hline \multirow{2}{*}{ Letras } & COM QUEIXA & \multirow{2}{*}{ G1 } & 0:00: 40 & 0:00: 18 & \multirow{2}{*}{0,10232} \\
\hline & SEM QUEIXA & & 0:00: 27 & 0:00: 04 & \\
\hline \multirow{2}{*}{ Dígitos } & COM QUEIXA & \multirow{2}{*}{ G1 } & 0:00: 42 & 0:00: 10 & \multirow{2}{*}{0,06307} \\
\hline & SEM QUEIXA & & 0:00: 34 & 0:00: 07 & \\
\hline \multirow{2}{*}{ Objetos } & COM QUEIXA & \multirow{2}{*}{ G1 } & 0:01: 12 & 0:00: 14 & \multirow{2}{*}{0,3618} \\
\hline & SEM QUEIXA & & 0:01: 06 & 0:00: 17 & \\
\hline & & Grupo & Média & Desvio padrão & Significância (P) \\
\hline \multirow{2}{*}{ Cores } & COM QUEIXA & \multirow{2}{*}{ G2 } & 0:01: 01 & 0:00: 45 & \multirow{2}{*}{0,32436} \\
\hline & SEM QUEIXA & & 0:00: 46 & 0:00: 07 & \\
\hline \multirow{2}{*}{ Letras } & COM QUEIXA & \multirow{2}{*}{ G2 } & 0:00: 41 & 0:00: 26 & \multirow{2}{*}{0,19571} \\
\hline & SEM QUEIXA & & 0:00: 29 & 0:00: 09 & \\
\hline \multirow{2}{*}{ Dígitos } & COM QUEIXA & \multirow{2}{*}{ G2 } & 0:00: 42 & 0:00: 30 & \multirow{2}{*}{0,27082} \\
\hline & SEM QUEIXA & & 0:00: 31 & 0:00: 06 & \\
\hline \multirow{3}{*}{ Objetos } & COM QUEIXA & \multirow{2}{*}{ G2 } & 0:01: 12 & 0:00: 33 & \multirow{2}{*}{0,5825} \\
\hline & SEM QUEIXA & & 0:01: 05 & 0:00: 15 & \\
\hline & & Grupo & Média & Desvio padrão & Significância $(P)$ \\
\hline \multirow{2}{*}{ Cores } & COM QUEIXA & \multirow{2}{*}{ G3 } & 0:00: 49 & 0:00: 09 & \multirow{2}{*}{0,0664} \\
\hline & SEM QUEIXA & & 0:00: 44 & 0:00: 07 & \\
\hline \multirow{2}{*}{ Letras } & COM QUEIXA & & 0:00: 34 & 0:00: 06 & \\
\hline & SEM QUEIXA & G3 & 0:00: 26 & 0:00: 05 & $0,00016^{*}$ \\
\hline & COM QUEIXA & & 0:00: 35 & 0:00: 07 & \\
\hline Digitos & SEM QUEIXA & G3 & 0:00: 29 & 0:00: 04 & $0,001692^{*}$ \\
\hline & COM QUEIXA & & $0: 01: 13$ & $0: 00: 23$ & \\
\hline Objetos & SEM QUEIXA & G3 & 0:00:59 & 0:00:12 & $0,02846^{*}$ \\
\hline & & Grupo & Média & Desvio padrão & Significância (P) \\
\hline & COM QUEIXA & & 0:00: 48 & 0:00: 06 & \\
\hline Cores & SEM QUEIXA & G4 & 0:00: 42 & 0:00: 09 & 0,068 \\
\hline & COM QUEIXA & & 0:00: 31 & 0:00: 04 & \\
\hline Letras & SEM QUEIXA & G4 & 0:00: 25 & 0:00: 09 & $0,007514^{*}$ \\
\hline & COM QUEIXA & & 0:00: 33 & 0:00: 04 & \\
\hline Dígitos & SEM QUEIXA & G4 & 0:00: 27 & 0:00: 07 & $0,0073958^{\star}$ \\
\hline & COM QUEIXA & & 0:01: 08 & 0:00: 07 & \\
\hline Objetos & SEM QUEIXA & G4 & 0:00: 57 & 0:00: 16 & $0,00806^{\star}$ \\
\hline
\end{tabular}

Significância $=p<0,05$

$\mathrm{G} 1=2^{\circ}$ ano $/ \mathrm{G} 2=3^{\circ}$ ano $/ \mathrm{G} 3=4^{\circ}$ ano $/ \mathrm{G} 4=5^{\circ}$ ano

pesquisada. Sujeitos considerados maus leitores ou que possuem alguma queixa relacionada à aprendizagem apresentam um pior desempenho em atividades que exijam velocidade de processamento de informações visuais $1,12,20,21$. Sabe-se que a aquisição da leitura e escrita está relacionada à velocidade de processamento de informações visuais. Uma vez que a rapidez é um fator importante para a leitura textual fluente, não é surpreendente que o desempenho de alunos com queixas de dificuldades de aprendizagem em tarefas de nomeação automatizada rápida tenha apresentado uma diferença estatisticamente significante, comparado aos alunos sem queixas. 
Tabela 3 - Distribuição da média, desvio-padrão e p-valor do desempenho geral de escolares com queixas aos sem queixas de transtornos de aprendizagem nos subtestes do teste de nomeação automática rápida (RAN)

\begin{tabular}{|c|c|c|c|c|}
\hline & Grupo & Média & Desvio padrão & Significância (P) \\
\hline \multirow{2}{*}{ Cores } & C/ QUEIXA & 0:00: 47 & 0:00: 09 & \multirow{2}{*}{0,563688854} \\
\hline & S/ QUEIXA & 0:00: 45 & 0:00: 08 & \\
\hline \multirow{2}{*}{ Letras } & C/ QUEIXA & 0:00: 31 & 0:00: 08 & \multirow{2}{*}{$0,010639264^{*}$} \\
\hline & S/ QUEIXA & 0:00: 26 & 0:00: 07 & \\
\hline \multirow{2}{*}{ Dígitos } & C/ QUEIXA & $0: 00: 34$ & 0:00: 06 & \multirow{2}{*}{$0,007365577^{*}$} \\
\hline & S/ QUEIXA & 0:00: 30 & 0:00: 07 & \\
\hline \multirow{2}{*}{ Objetos } & C/ QUEIXA & 0:01: 04 & 0:00: 10 & \multirow{2}{*}{0,211264411} \\
\hline & S/ QUEIXA & 0:01: 01 & 0:00: 15 & \\
\hline
\end{tabular}

Significância $=p<0,05$

Tabela 4 - Distribuição da média, desvio-padrão e p-valor do desempenho geral de escolares do sexo masculino comparado ao feminino nos subtestes do teste de nomeação automática rápida (RAN)

\begin{tabular}{|c|c|c|c|c|}
\hline & Sexo & Média & Desvio padrão & Significância $(P)$ \\
\hline \multirow{2}{*}{ Cores } & FEMININO & 0:00: 48 & 0:00: 17 & \multirow{2}{*}{0,373859332} \\
\hline & MASCULINO & 0:00: 46 & 0:00: 10 & \\
\hline \multirow{2}{*}{ Letras } & FEMININO & 0:00: 29 & 0:00: 11 & \multirow{2}{*}{0,770039497} \\
\hline & MASCULINO & 0:00: 29 & 0:00: 10 & \\
\hline \multirow{2}{*}{ Dígitos } & FEMININO & 0:00: 32 & 0:00: 12 & \multirow{2}{*}{0,699485638} \\
\hline & MASCULINO & 0:00: 32 & 0:00: 08 & \\
\hline \multirow{2}{*}{ Objetos } & FEMININO & 0:01: 05 & 0:00: 20 & \multirow{2}{*}{0,472712085} \\
\hline & MASCULINO & 0:01: 03 & 0:00: 16 & \\
\hline
\end{tabular}

Significância $=p<0,05$

Tabela 5 - Velocidade / tempo mínimo e máximo obtidos pelos sujeitos sem queixas de problemas de aprendizagem dos grupos G1, G2, G3 e G4

\begin{tabular}{ccccccccc}
\hline & \multicolumn{2}{c}{ G1 } & \multicolumn{2}{c}{ G2 } & \multicolumn{2}{c}{ G3 } & \multicolumn{2}{c}{ G4 } \\
\cline { 2 - 8 } & Mínimo & Máximo & Mínimo & Máximo & Mínimo & Máximo & Mínimo & Máximo \\
\hline Cores & $00: 00: 40$ & $0: 01: 05$ & $0: 00: 30$ & $0: 00: 59$ & $0: 00: 34$ & $0: 00: 58$ & $0: 00: 29$ & $0: 00: 56$ \\
Letras & $00: 00: 20$ & $0: 00: 37$ & $0: 00: 22$ & $0: 01: 00$ & $0: 00: 19$ & $0: 00: 36$ & $0: 00: 16$ & $0: 00: 39$ \\
Dígitos & $00: 00: 22$ & $00: 00: 45$ & $0: 00: 22$ & $0: 00: 45$ & $0: 00: 22$ & $0: 00: 38$ & $0: 00: 17$ & $0: 00: 44$ \\
Objetos & $00: 00: 41$ & $00: 01: 53$ & $0: 00: 47$ & $0: 01: 18$ & $0: 00: 44$ & $0: 01: 28$ & $0: 00: 34$ & $0: 01: 42$ \\
\hline
\end{tabular}

$\mathrm{G} 1=2^{\circ}$ ano $/ \mathrm{G} 2=3^{\circ}$ ano $/ \mathrm{G} 3=4^{\circ}$ ano $/ \mathrm{G} 4=5^{\circ}$ ano

O aumento da idade e grau de escolaridade acarreta um aumento da capacidade linguísticocognitiva do sujeito aumentando, portanto, o nível de exigência escolar sobre este indivíduo ${ }^{10,18,20}$. O fato de crianças com problemas de aprendizagem apresentarem desempenho inferior aos seus pares sem queixas de problemas de aprendizagem fica mais evidente nas séries mais avançadas, na qual o grupo já deveria apresentar uma maturação do processamento geral de velocidade, correspondendo assim às exigências do contexto escolar ${ }^{20}$.

Com frequência são os pais e professores que detectam inicialmente o problema de aprendizagem da criança ${ }^{22}$. Todas as crianças indicadas como 
apresentando queixa de problemas de aprendizagem pelos pais, foram confirmadas pelo questionário dos professores como maus leitores. Tais dados nos mostram que pais e professores têm condições de perceber precocemente a dificuldade da criança e devem procurar ajuda, para que ocorra o mais brevemente possível o diagnóstico de um possível problema de aprendizagem, evitando transtornos futuros no rendimento escolar.

Os códigos letras e dígitos foram nomeados com maior rapidez e facilidade do que as cores e os objetos ${ }^{23}$. A nomeação dos objetos é significativamente mais lenta que os demais códigos, seguidos pelas cores, corroborando os achados da literatura ${ }^{1,7,10}$. Este achado pode ser explicado pelo fato de a nomeação de figuras requerer sempre o acesso ao significado para a posterior produção do nome. Por outro lado, a leitura de palavras pode ser realizada sem passar por este processo, ou seja, para a identificação de um grafema ou dígito não há necessidade de acesso ao significado ${ }^{24}$. Objetos e cores apresentam uma carga semântica maior.

Ao analisar os tempos mínimos e máximos obtidos por sujeitos considerados proficientes, sem queixas de problemas de aprendizagem, obteve-se uma possível média esperada para a população mineira, que se encontra muito próxima às médias encontradas na população paulista, pesquisada em $2003{ }^{10}$, com mesma idade e escolaridade. Após uma análise estatística comparando o tempo de nomeação dos escolares paulistas com o dos mineiros, não foram observadas diferenças estatisticamente significantes. Isso revela que o tempo de nomeação não está relacionado a variações linguísticas, como velocidade de fala e prosódia. A nomeação automatizada relaciona-se a velocidade de acesso ao léxico e ao processamento de estímulos visuais.

\section{CONCLUSÃO}

Por meio desta pesquisa pode-se concluir que o desempenho no teste de nomeação rápida automatizada (RAN) de escolares com queixas de dificuldades de aprendizagem foi significativamente inferior ao de escolares sem histórico de dificuldade de aprendizagem. Da mesma forma foi possível concluir que os escolares de rede pública de ensino apresentaram pior desempenho quando comparados aos escolares da rede privada.

O presente estudo demonstrou que a habilidade de nomeação seriada pode estar relacionada ao desempenho de leitura. Sendo assim, há necessidade de continuidade de pesquisas que estabeleçam a relação desta habilidade da linguagem com outras habilidades como leitura e escrita, consciência fonológica, memória de trabalho, nas populações aqui analisadas para verificação do impacto direto desta habilidade no processo de aprendizagem da leitura.

\begin{abstract}
Purpose: to investigate rapid serial naming of children in a public and private school- with and without complaints of school problems. Methods: 138 children of both genders took part in the study, with age ranging between 7 and 11 years, of the city of Belo Horizonte, fundamental teaching, between the 2nd and the 5th year. The Test of- Rapid Automated Naming - (RAN) was applied and we evaluated the time spend by the subject to name a series of family visual incentives: colors, letters, digits and objects - as fast as possible. Results: the results of this study evidenced that the private school acting of students compared to that of the students in public school is superior and that there is a- significant statistical difference- among subjects with complaints of learning problems as compared to those without complaints. Conclusion: this study signals that the capacity of serial nomination can be considered as one of the fundamental abilities for the good acting in reading. So, there is a need for continuity of researches that will set up the relationship of the ability in the language with other reading- and writing abilities for checking the direct impact of the fast nomination in the process of reading learning.
\end{abstract}

KEYWORDS: Learning; Child; Reading; Education; Velocity Measurement 


\section{REFERÊNCIAS}

1. Salgado CA, Capellini SA. Programa de remediação fonológica em escolares com dislexia do desenvolvimento. Pró-Fono. 2008; 20(1):31-6.

2. Teles P. Dislexia: como identificar? Como intervir? Rev Port Clin Geral. 2004; 20(5):13-30.

3. Schirmer CR, Fontoura DR, Nunes ML. Distúrbios da aquisição da linguagem e da aprendizagem. J Pediatr. 2004; 80(Supl2):S95-S102.

4. Gindri G, Keske-Soares M, Mota HB. Memória de trabalho, consciência fonológica e hipótese de escrita. Pró-Fono. 2007; 19(3):313-22.

5. Georgiou GK, Das JP, Hayward DV. Comparing the contribution of two tests of working memory to reading in relation to phonological awareness and rapid naming speed. J Res Read. 2008; 31(3):302-18.

6. Capellini AS, Ferreira TL, Salgado CA, Ciasca SM. Desempenho de escolares bons leitores, com dislexia e com transtorno do déficit de atenção e hiperatividade em nomeação automática rápida. Rev Soc Bras Fonoaudiol. 2007; 12(2):114-9.

7. Denckla MB, Rudel R. Rapid "automatized" naming of pictured objects, colors, letters and numbers by normal children. Cortex. 1974; 10(2):186-202.

8. Shaywitz SE, Shaywitz BA, Pugh KR, Fulbright RK, Constable RT, Mencl WE, et al. Functional disruption in the organization of the brain for reading in dyslexia. Proc Natl Acad Sci USA. 1998; 95(5):2636-41.

9. Santos MTM. Vocabulário, consciência fonológica e nomeação rápida: contribuições para a ortografia e elaboração escrita. Rev Soc Bras Fonoaudiol. 2007; 12(3):262.

10. Ferreira TL, Capellini SA, Ciasca SM, Tonelotto JMF. Desempenho de escolares leitores proficientes no teste de nomeação automatizada rápida - RAN. Temas Desenvolv. 2003; 12(69):26-32.

11. Stivanin L, Scheuer $\mathrm{Cl}$. Tempo de latência e exatidão para leitura e nomeação em crianças escolares: estudo piloto. Educ Pesq. 2005; 31(3):425-36.

RECEBIDO EM: 22/04/2009

ACEITO EM: 17/11/2009

Endereço para correspondência:

Lorena Gabrielle Ribeiro Bicalho

Rua Nossa Senhora do Brasil, 173

Belo Horizonte - MG

CEP: 31130-090

E-mail: Iorena.bicalho@ hotmail.com
12. Cardoso-Martins C, Pennington B. Qual é a contribuição da nomeação seriada rápida para a habilidade de leitura e escrita? Evidência de crianças e adolescentes com e sem dificuldades de leitura. Psicol Reflex Crit. 2001; 14(2):387-97.

13. Santos MTM. Terapia do processamento ortográfico. In: Santos MTM, Navas ALG. Distúrbios de leitura e escrita. São Paulo: Manole; 2002. p.329-49.

14. Howe AE, Arnell KM, Klein RM, Joanisse MF, Tannock R. The ABCs of computerized naming: equivalency, reliability, and predictive validity of a computerized rapid automatized naming (RAN) task. J Neuroscie Methods. 2006; 151(1):30-7.

15. Fellipe ACN, Colafêmina JF. Avaliação simplificada do processamento auditivo e o desempenho em tarefas de leitura-escrita. PróFono. 2002; 14(2):225-34.

16. Pinheiro AMV, Costa AEB. Escala de avaliação de competência em leitura pelo professor. Resumo publicado no VII Encontro Mineiro de Avaliação Psicológica, Belo Horizonte, março de 2005.

17. Ciasca SM. Distúrbios de aprendizagem: proposta de avaliação interdisciplinar. São Paulo: Casa do Psicólogo; 2003. p. 28.

18. Simões VF. Estudo do desempenho de crianças das séries iniciais: do ensino fundamental I em testes de leitura, escrita e nomeação rápida [tese]. São Paulo (SP): Universidade de São Paulo; 2006. 19. Demo P. Escola pública e escola particular: semelhanças de dois imbróglios educacionais. Ensaio: Aval Pol Públ Educ. 2007; 15(55):181-206. 20. González MRO, Hernández-Valle I, Monzón AE, López MR, Guzmán R, García E, et al. Evaluación de la velocidad de nombrar en las dificultades de aprendizaje de la lectura. Psicothema. 2004; 16(3):442-7.

21. Capellini, AS, Conrado TLBC. Desempenho de escolares com e sem dificuldades de aprendizagem de ensino particular em habilidade fonológica, nomeação rápida, leitura e escrita. Rev. CEFAC. 2009; 11(Supl2):183-93. http://dx.doi.org/10.1590/ S1516-18462009005000002

22. ahead of print

23. Zucoloto KA. A compreensão da leitura em crianças com dificuldades de aprendizagem na escrita. [dissertação]. Campinas (SP): Universidade Estadual de Campinas; 2001.

24. Mousinho R, Correa J. Habilidades lingüísticocognitivas em leitores e não-leitores. Pró-Fono. 2009; 21(2):113-8.

25. Stivanin L, Scheuer C. Comparação do tempo de latência entre nomeação e leitura em escolares. Psicol Estud. 2008; 13(1):89-96. 